\title{
Conocimiento, Internet y democracia
}

Knowledge, Internet and democracy

\author{
Fernando GALINDO AYUDA \\ Facultad de Derecho, Universidad de Zaragoza, Pedro Cerbuna 12, 50009 Zaragoza, España \\ cfa@unizar.es
}

\begin{abstract}
Resumen
Se presenta una reflexión sobre el alcance y los límites que tienen, desde una perspectiva jurídica, las prácticas democráticas en Internet. Se atiende a las características de algunas experiencias que promueven la participación de usuarios de Internet en la actividad pública mediante el conocimiento que les proporciona sobre las actividades de las instituciones políticas.
\end{abstract}

Palabras clave: Conocimiento. Internet. Democracia.

\section{Introducción}

La expresión democracia aparece reiteradamente en Internet. Y ello no sólo en lo relativo a la acumulación de información sustantiva, de contenido -es decir definiciones, expresiones, teorías y conceptos, que sobre democracia se hace en la red, entendiéndola como término que ayuda a caracterizar a aquellos regímenes políticos en los que los ciudadanos eligen a sus representantes y con ello participan en el proceso de gobierno público-, sino también en lo que se refiere a la relación existente, en muchas ocasiones equiparación, entre Internet y democracia, entendiendo que el funcionamiento de la misma Internet o la "red" es democrático o propicia la democracia, al considerar que facilita a todo el que la maneja -en definitiva, al que cuenta con un medio de acceso a la misma (ordenador, teléfono móvil o televisor interactivo, por ejemplo) - un conocimiento / poder / dominio sobre la realidad que antes no tenía: Estaba reservado a quien había sido nombrado "gobernante" en el proceso de elección democrática de representantes políticos. En el presente trabajo discurrimos sobre estas cuestiones centrándonos, especialmente, en el segundo de los aspectos, contrastándolo con el primero, es decir averiguando la efectiva potenciación de la democracia, entendida como participación política, que genera el uso de Internet.

A estos efectos prestamos, en primer lugar, atención a determinadas iniciativas que potencian una efectiva realización de la democracia mediante el uso de Internet, asumiendo que democracia es también participación de ciuda-

\begin{abstract}
A reflection on the scope and limits that the democratic practices have in Internet is presented from a legal perspective. The characteristics of some experiences that promote the participation of Internet users in the public activities are analyzed, with a focus on the knowledge that Internet provides on the activities of political institutions.
\end{abstract}

Keywords: Knowledge. Democracy. Internet.

danos informados en la actividad pública. En segundo lugar, mostramos algunos ejemplos reales de potenciación de la actividad de las instituciones democráticas que se ponen en práctica mediante el uso de las TIC e Internet. En tercer lugar, resumimos consideraciones generalmente aceptadas sobre democracia y participación política que se producen en la actualidad, a efectos de comprender las implicaciones políticas y los límites de los fenómenos estudiados en este trabajo. En cuarto lugar, concluimos.

\section{Conocimiento}

\subsection{Internet e información}

Un requisito fundamental para el ejercicio de la democracia es contar con información suficiente para decidir. Ello es coherente con el hecho de que sin información no es posible tomar decisión alguna (Maturana, 2006). Si esto es así en las actividades propias -en el ejercicio de la libertad o del principio de autonomía de la voluntad-, mayor relevancia tiene en el ámbito de acción de los hombres referido a las actividades que implican a otros hombres. También en la acción política y el ejercicio democrático de la libre elección de los representantes políticos: No se puede elegir sin conocer previamente las opciones sobre las que cabe optar.

E Internet proporciona, sin duda, acceso a información. Ofrece una información que antes, sin ella, no estaba al alcance de los ciudadanos. La información quedaba, a lo sumo, recogida en documentos: estudios, informes, artículos cientí- 
ficos y de opinión, libros y noticias suministradas por los medios de publicación, opinión e información —periódicos, revistas y otros medios (televisión y radio fundamentalmente). Otra parte de la información y especialmente la referida a las actividades del Estado quedaba fuera de los círculos de opinión. Con gran frecuencia se consideraba secreta: estaba a disposición únicamente de las instituciones estatales.

La información necesaria para elegir democráticamente es la referida a las actividades de los representantes políticos, cuya expresión se manifiesta en la acción propia de las instituciones en las que los mismos las realizan. Son las actividades de los poderes legislativo, ejecutivo y judicial. Están recogidas en las leyes, normas, reglamentaciones, sentencias y, ahora, también en las páginas web en las que se presenta tanto el contenido de las regulaciones cuanto que informaciones sobre las características y resultados de las actividades desenvueltas y los servicios públicos ofertados por cada institución.

La información relativa al contenido de las regulaciones es la misma, sobre formato digital, que la que se hacía pública en papel. La principal diferencia resulta del grado de expansión de la información digital: es mayor que la que tiene la recogida en formato papel. La información sobre las instituciones, sus actividades y servicios, es de diferente entidad: antes no era accesible, no existía o a lo sumo encontraba algún eco en los medios de comunicación o, con el tiempo, en los trabajos realizados por los historiadores. En la mayor parte de las ocasiones estaba expresada en el contenido de los propios textos jurídicos o regulaciones. En la actualidad esta información es generada por la propia institución, que puede ofrecer sus servicios a los ciudadanos a través de Internet.

Esta información precisa ser expuesta, publicitada y recopilada de forma ordenada y suficientemente clara si se quiere que los ciudadanos puedan acceder a la misma, utilizar los servicios públicos y realizar el control democrático de la misma que compete a los ciudadanos. Es preciso, también, establecer algunas indicaciones para efectuar adecuadamente esta recopilación, atendiendo tanto al contenido de las prácticas desarrolladas como a lo que prescriben las reglas de la democracia encaminadas a potenciar la participación de los ciudadanos en el poder político.

\subsection{Datos públicos}

De todo lo anterior se ocupan iniciativas que tienen por objeto hacer llegar a los ciudadanos los datos o la información pública que se encuentra en Internet (1).

La más mínima observación sobre la información recopilada por este tipo de iniciativas da cuenta de que responde a fines $u$ objetivos dispares: ofertar la información a elaboradores de estudios, proporcionar acceso seguro a servicios públicos, mostrar el alcance de las iniciativas propias de la entidad administrativa responsable de la página o sitio web, señalar los avances de la técnica, poner en funcionamiento el principio legal de transparencia de las actividades gubernamentales, mostrar el grado de eficiencia de políticas de gobernanza, generar actividades a desarrollar por empresas y con ello crear empleo... Todo lo cual trae como consecuencia la existencia de numerosa información en Internet situada, todavía, en forma escasamente accesible al ciudadano, una vez que éste no cuenta con suficientes conocimientos ni recursos como para poder acceder a la información ni, una vez accedida, comprenderla. Ello, por tanto, no se debe únicamente al limitado porcentaje de personas que acceden a la red, la brecha digital, sino al hecho de que la información está colocada con gran frecuencia en lenguaje propio de profesionales expertos en contabilidad, por ejemplo, pero no en lenguaje comprensible por los ciudadanos.

Es por ello que de un tiempo a esta parte se producen iniciativas que tienen el objetivo de promover la participación ciudadana en forma activa en el ejercicio de sus derechos democráticos, haciendo accesible mediante Internet a los ciudadanos no sólo los datos generados por los gobiernos sino el mismo funcionamiento y actividades de las Administraciones públicas y de los diferentes poderes públicos (2). A esos efectos se utilizan aproximaciones y recursos tecnológicos que ponen mayor énfasis en la promoción de la participación democrática y la inclusión digital que la implantación del propio desarrollo tecnológico a través de la democracia electrónica, del que se ocupan otro tipo de investigaciones (3).

Un paso en este marco de acción es el constituido por las investigaciones y desarrollos que desde 2003 se realizan por el Observatorio Internacional de Gobierno electrónico.

\subsection{El Observatorio EGOBS}

EGOBS (Electronic Government Observatory, www.egobs.org) es una iniciativa surgida de la red temática Gobierno Electrónico coordinada por la Universidad de Zaragoza (España) y en cuyas actividades participan desde 2003 las Universidades de Burgos y Valladolid (España), 
la Universidad de Münster (Alemania) y la Queen's University de Belfast (Reino Unido), por parte europea. Los miembros americanos son la Universidad Nacional de la Plata (Argentina), la Universidad Diego Portales de Santiago de Chile, la Universidad de la Habana (Cuba) y la Universidad de la República de Montevideo (Uruguay). Colabora con las actividades del Observatorio la Asociación para la Promoción de las Tecnologías de la Información y el Comercio Electrónico (APTICE). El Observatorio es parte de la Red Jurídica para la Sociedad de la Información LEFIS (Legal Framework for the Information Society).

Desde el 1 de enero de 2009 el Observatorio cuenta con infraestructura personal y material para el desarrollo de sus actividades en Iberoamérica. En concreto en la Universidad Federal de Santa Catalina (Brasil) (4).

El objetivo fundamental del Observatorio EGOBS es el estudio independiente de las características de las realizaciones que en materia de Administración / Gobierno electrónico tienen lugar en distintos países, esto es el conjunto de organizaciones y sistemas que son precisos para que se produzca la prestación de servicios administrativos a ciudadanos y empresas por las Administraciones Públicas con auxilio de los recursos que facilitan las Tecnologías de la Información y la Comunicación.

\subsection{Metodología LEFIS}

Los trabajos realizados se preocupan por la transferencia de conocimientos: ponen especial énfasis en la comprobación referida a si la prestación se realiza con respeto a las normas, procedimientos y principios recogidos en las leyes -incluido el derecho a la participación en dichas actividades de ciudadanos, empresas e instituciones- $y$, especialmente, si se satisfacen en su puesta en práctica las regulaciones sobre protección de datos y seguridad de las comunicaciones electrónicas.

Las actividades de observación de EGOBS han estado centradas en aplicar, con fines docentes e investigadores la metodología LEFIS (Galindo, García, Lasala, 2006) desarrollada para estudiar páginas web, confeccionadas e implementadas por Administraciones públicas, utilizando criterios de análisis que permiten conocer y presentar públicamente las características de las propias páginas, sus contenidos y el de las ofertas de servicios que realizan las Administraciones que las han confeccionado y mantienen (5).

La metodología se ha venido aplicando desde el año 2005 a páginas web desarrolladas por insti- tuciones públicas en Chile, Uruguay, España y Brasil, fundamentalmente. Los resultados de los análisis están expuestos en Internet (6).

\subsection{Información}

A partir de 2009 EGOBS realiza otro tipo de análisis destinado a suministrar información: presentar en Internet a ciudadanos en forma clara la localización geográfica de instituciones públicas que realizan distintas actividades. Este tipo de información se refiere básicamente a instituciones brasileñas. El interés básico de este tipo de análisis es el fomento de la inclusión digital.

Los estudios se expresan en forma de páginas web que contienen mapas de Brasil en los que se recoge la localización de instituciones y actividades realizadas por las mismas en relación a materias concretas (7).

La lista de materias en forma de temas generales en la actualidad es la siguiente: medio ambiente; cultura y educación pública; democracia, convergencia e inclusión digital; hacienda pública, poder judicial, ministerio público, modelos y proyectos de gobierno electrónico, salud pública y seguridad pública.

\subsection{Mapas}

En relación a cada tema se presentan varios mapas de ámbito federal o estatal, en los cuales, por ejemplo, se localiza la situación de instituciones, centros de estudio, actividades realizadas, distribución de recursos públicos con respecto a proyectos concretos y sitios web analizados siguiendo la metodología LEFIS.

Los mapas son resultados de investigaciones realizadas bajo la responsabilidad de investigadores concretos que elaboran trabajos o artículos sobre la respectiva materia. Pretenden crear discusión, a la vez que, fundamentalmente, ilustrar sobre las materias estudiadas a ciudadanos que no tengan formación especializada sino simplemente interés por conocer cuestiones concretas a efectos de crear opinión política. En este sentido son instrumentos tecnológicos de transferencia de información y conocimiento que fomentan la democracia.

\section{TIC y democracia}

Además de proporcionar información y conocimiento $-\mathrm{y}$ por lo mismo incrementar la democracia-, no cabe duda de que las Tecnologías de la Información y la Comunicación (TIC) e Internet facilitan la puesta en funcionamiento de los sistemas políticos democráticos. Aquí vamos 
a presentar tres ejemplos que lo prueban inequívocamente. Uno está referido al auxilio a la elección de los representantes políticos. Otro se refiere al acceso de los ciudadanos a los servicios públicos por medios electrónicos. El último se ocupa del apoyo al funcionamiento del poder judicial. A continuación resumimos las características básicas de los ejemplos.

\subsection{Urnas electrónicas}

El primer ejemplo tiene manifestaciones masivas fundamentalmente en Brasil. Desde la segunda mitad de los años noventa los procesos electorales destinados al nombramiento de los representantes políticos se auxilian de las denominadas "urnas electrónicas". Son programas y ordenadores que facilitan que el derecho de voto con el fin de elegir a sus representantes políticos que tienen todos los ciudadanos sea ejercido por los electores de los candidatos mediante la pulsación, autorizada por los integrantes de la mesa de votación, a los electores que son identificados mediante la aportación de un documento, del número correspondiente, la denominación, asignado a los candidatos a ser elegidos. El programa almacena las opciones tomadas por los electores y suma los resultados finales de la votación. De esta forma el uso de sistemas tecnológicos mejora el ejercicio del voto al dificultar la realización de prácticas corruptas electorales que en Brasil permitían los sistemas tradicionales de elección política, promoviendo con ello el uso de las TIC la ampliación y profundización de la democracia (Mezzaroba y Rover, 2009).

\subsection{Administración electrónica}

El segundo ejemplo está extendido por muchos países. Es el denominado Gobierno electrónico o, más adecuadamente, Administración electrónica (8). Hace referencia a la provisión por medios electrónicos de acceso de los ciudadanos a los servicios públicos. Implica que los ciudadanos puedan tramitar por Internet desde sus domicilios o sus propios ordenadores instancias a las Administraciones Públicas sin necesidad de realizar desplazamientos a la sede de las Administraciones de las que se requiera la satisfacción de un derecho concreto: un certificado, un servicio, una subvención, una licencia.... El proceso tiene lugar de la siguiente forma. Una vez identificado fidedignamente el solicitante mediante la utilización de una clave obtenida usando un procedimiento seguro, el ciudadano interesado -el solicitante- puede remitir por Internet su solicitud, junto al expediente que la justifica o fundamenta, al ordenador-programa de la Administración, la sede electrónica, cuya sección administrativa competente tenga la obligación de tramitar el expediente reconociendo lo requerido por el solicitante. El sistema tecnológico, las TIC, puede mejorar, por tanto, la calidad democrática de la Administración correspondiente, al incrementar la prontitud de respuesta de los servicios que la integran, potenciando de esta forma la satisfacción de los derechos de los ciudadanos (9).

\subsection{Proceso judicial electrónico}

El tercer ejemplo — LEXNET (10)_ está referido al ámbito judicial y sucede en varios países. Un abogado, convenientemente identificado, puede enviar a un Juzgado o Tribunal desde cualquier ordenador un documento que forme parte de un proceso en el que esté implicado un ciudadano defendido (o acusado) por el abogado, a efectos de que el trámite procesal se dé por realizado, pudiéndose con ello continuar su tramitación en un periodo de tiempo menor que el que es preciso para que el procedimiento discurra por los canales establecidos para la tramitación del documento en formato papel al evitarse las dilaciones propias del funcionamiento del correo ordinario. El sistema, además, permite a todas las partes implicadas en el proceso comprobar el estado de tramitación del procedimiento, pudiéndose conocer tanto las fases del proceso satisfechas cuanto el contenido de los documentos presentados por la otra parte o las resoluciones o diligencias adoptadas por el órgano instructor o juzgador. Las TIC favorecen también en este caso a la democracia una vez que el procedimiento que facilitan permite tanto acortar los tiempos precisos para el ejercicio de las labores propias del poder judicial: la resolución de conflictos, cuanto hacer más transparentes las fases de tramitación del procedimiento, permitiendo conocer por las partes implicadas en el caso el estado en el que la tramitación se encuentra, sin que por ello pierda el proceso judicial las funciones conferidas al mismo por el ordenamiento democrático.

No cabe duda de que estos procesos son ejemplos de que las TIC son efectivos instrumentos auxiliares del funcionamiento de los sistemas democráticos.

Pero ¿qué es democracia?

\section{Democracia hoy}

Un sistema político democrático es el que está organizado atendiendo a la garantía e impulso de tres mecanismos, hoy principios jurídicos fundamentales, reconocidos en las constituciones y hechos realidad en la vida diaria de los países donde las mismas funcionan. Son los 
referidos a la libre elección de los gobernantes por los ciudadanos, la efectiva puesta en práctica de la división del ejercicio del poder político por las instancias de gobierno competentes, y la salvaguarda de la protección y promoción (Carbonell, 2008) de los derechos humanos por los poderes públicos con respecto a las actividades que tienen lugar en el desarrollo de la vida propia de los ciudadanos, de los ciudadanos con las empresas como consumidores o trabajadores o empleados, de las empresas entre sí, de los ciudadanos con otros ciudadanos, de las administraciones públicas con otras administraciones, de las administraciones públicas con las empresas y a la inversa, y de los ciudadanos con las administraciones públicas y a la inversa en relación a la prestación por las últimas de servicios públicos.

Los tres mecanismos son considerados propios del funcionamiento del Estado de Derecho. Su contenido y función característicos son resumidos a continuación.

El primer mecanismo es el de la libre elección de los gobernantes por los ciudadanos, que se pone en práctica mediante la realización de procesos electorales que permiten a todos los ciudadanos participar en el gobierno nombrando periódicamente a sus representantes en distintos ámbitos políticos de decisión: estatal, regional/autonómico o local. Este procedimiento participativo, democrático, se ve completado por la posibilidad que tienen los ciudadanos de intervenir en las actividades gubernamentales mediante la puesta en realidad de otros medios como el referéndum con relación a cuestiones concretas sometidas a opinión de la ciudadanía por los representantes políticos, la coparticipación en actividades de carácter gubernamental mediante la intervención de particulares o ciudadanos, organizaciones, asociaciones ciudadanas, asociaciones de consumidores o sindicatos, cuya actuación está prevista en la regulación de procedimientos administrativos, y la participación de los ciudadanos en la solución de conflictos judiciales mediante su intervención como jurados.

El segundo mecanismo democrático es la división de poderes, es decir la aceptación de que el ejercicio de los recursos, instrumentos y medios que han de poner en práctica los gobernantes elegidos, tiene que atender a la separación de dicho ejercicio en el de tres "poderes" o funciones: legislativo, ejecutivo y judicial. Ello implica que la actividad propia de cada poder ha de ser realizada respetando la distribución de las competencias funcionales establecida mediante la satisfacción de los procedimientos reconocidos y articulados por la constitución y el resto del ordenamiento. Estos procedimientos permiten garantizar tanto la independencia de acción de cada uno de los poderes, cuanto que la coordinación y el equilibrio del ejercicio de las funciones o competencias propias de los mismos.

El tercer mecanismo es el del reconocimiento, respeto, preservación y promoción de las declaraciones de derechos humanos contenidas en la constitución y el ordenamiento jurídico en su conjunto, realizados por la práctica del ejercicio de las competencias y funciones propias de los poderes con respecto a actividades ocurridas en sociedades concretas.

Completa lo hasta aquí expresado la consideración de que, obviamente, la democracia o la participación política no puede ser puesta en práctica efectivamente en ninguna de las facetas reseñadas sin la satisfacción de un requisito previo: que los ciudadanos estén informados 0 , lo que es lo mismo, tengan suficiente conocimiento sobre el objeto de su participación. Sea ello, por ejemplo, las características de las actividades administrativas, el efectivo cumplimiento de las subvenciones otorgadas por la Administración con el fin de satisfacer un concreto objetivo político como la dotación de Internet a unas escuelas, el programa político de un candidato a ser elegido como parlamentario o como concejal, el cumplimiento del programa político de un representante político que vuelve a presentarse a las elecciones, el contenido de la decisión en una propuesta de referéndum o decisión administrativa, o las características de un caso sobre el que el ciudadano en cuestión tenga que resolver como integrante de un jurado.

Es por ello que en la actualidad cabe decir, sintéticamente, que un sistema político democrático es aquel cuyo funcionamiento está basado en la participación consciente de los ciudadanos en el ejercicio del poder político o bien indirectamente mediante la elección de sus representantes o bien directamente colaborando en la toma de decisiones políticas utilizando otros mecanismos. Todo lo cual implica reconocer que los ciudadanos pueden participar, por si o mediante sus representantes, en prácticamente todas las actividades de los poderes públicos, atendiendo, además, al hecho de que el Estado de Derecho actual no es el Estado liberal del siglo XIX que limitaba la acción de los organismos públicos a actuar políticamente como policía elaborando las correspondientes leyes básicas y aplicándolas mediante penas o sanciones a los infractores más graves del orden jurídico: salvaguardando el funcionamiento del mercado, sus posibles violaciones, sino que el Estado es, 
a la vez, Estado social, democrático, del bienestar, de la gobernanza y, hoy, el Estado propio de la denominada sociedad del conocimiento o de las TIC, que tiene potestad para participar en prácticamente todas las actividades diarias especialmente las propias de las instituciones públicas una vez están financiadas con fondos públicos.

\subsection{Gobernanza}

Lo dicho hasta aquí no impide el reconocimiento de la que de un tiempo a esta parte se está aceptando como práctica política propia común de las competencias propias de los poderes públicos: la del ejercicio de lo que se denomina gobernanza. La gobernanza está definida por el Diccionario como "Arte o manera de gobernar que se propone como objetivo el logro de un desarrollo económico, social e institucional duradero, promoviendo un sano equilibrio entre el Estado, la sociedad civil y el mercado de la economía".

Lo anterior implica reconocer la expansión en el ámbito público, como prácticas o usos propios de los gobernantes, de los principios, técnicas o usos de gobierno propios del ámbito empresarial. Esto es lo mismo que decir: la puesta en acción de la eficiencia y las reglas del mercado como criterio de acción preferente de los poderes públicos.

Este estilo de acción o política ha de ser ejercido por los poderes en forma compatible con la puesta en práctica de los principios propios del Estado de Derecho, que resumen la acción de la democracia que, por mandato legal, gobierna la acción de los poderes públicos, es decir todos aquellos asuntos sobre los que éstos son competentes según el ordenamiento propio de los Estados de Derecho en cuanto son agentes activos en la vida social de la sociedad del conocimiento (Katz, 2010; Galindo 2007).

A lo dicho hay que añadir que las reglas de funcionamiento de Internet son las propias del ámbito empresarial, lo que produce distorsiones en sus aplicaciones en el ámbito público (Christou y Simpson, 2009) y, por supuesto, en el fomento a la democracia que proporciona.

Es por todo lo anterior que podemos llegar a la conclusión.

\section{Conclusión}

Las expuestas en este trabajo son diferentes formas, concretas, de crear democracia mediante Internet; y, por ello, son distintas posibilidades de incrementar la democracia. Las posibilidades muestran que -aunque la democracia en Inter- net siempre estará limitada por el funcionamiento inevitablemente "interesado" de la gestión de Internet- hay margen para incrementar la democracia entendida como participación ciudadana en el conocimiento de las actividades públicas propiciando al mismo tiempo la inclusión digital utilizando los recursos que contiene Internet.

\section{Agradecimientos}

Este trabajo se desarrolla en el marco de los siguientes proyectos: Establecimiento en Iberoamérica del Observatorio de Gobierno Electrónico. EGOBS, Acción integrada para el fortalecimiento institucional, financiada por la Agencia Española para la Cooperación Internacional al Desarrollo (AECID), 2009-2012; y La nueva ecología de la información y la documentación en la sociedad del conocimiento: desarrollo de una métrica sistémica, planificación de un observatorio para su seguimiento e identificación de tendencias básicas y retos estratégicos (infoscopos.com), proyecto financiado por la CICYT 2010-12 (CSO200907619).

\section{Notas}

(1) Un catálogo sobre datos administrativos públicos recogidos por distintas instituciones gubernamentales se encuentra en: http://eadminblog.net/post/2010/04/11/listade-catalogos-de-open-data-en-el-mundo (15-3-2011). Elaborados por el Gobierno del País Vasco: http://open data.euskadi.net/w79-home/es (15-3-2011). Sobre actividades administrativas del Gobierno del Reino Unido: http://data.gov.uk/ (15-3-2011). La Funda-ción sobre datos abiertos, organización no lucrativa, promueve el uso de datos estadísticos: http://www.opendatafounda tion.org/ (15-3-2011).

(2) Directiva europea sobre transparencia: Directiva 2003/98/CE del Parlamento Europeo y del Consejo de 17 de noviembre de 2003 relativa a la reutilización de la información del sector público. Art. 1.1: "La presente Directiva establece un conjunto mínimo de normas que regulen la reutilización y los instrumentos prácticos que faciliten la reutiliza-ción de los documentos existentes conservados por organismos del sector público de los Estados miembros". http://eur-lex.europa.eu/LexUriServ/ LexUriServ.do?uri=CELEX:32003L0098:ES:HTML (153-2011). En España, el proyecto Aporta (www.aporta.es) "promueve una cultura de reutilización de la información en el ámbito de la Administración pública, concienciando de la importancia y el valor que tiene la información del sector público y su reutilización, y quiere facilitar la puesta a disposición por parte de las Administraciones y organismos públicos de la información disponible, aprovechando así el potencial del mercado de reutilización de la información pública en España." (15-3-2011).

(3) Por ejemplo, http://forums.e-democracy.org (15-3-2011).

(4) http://www.egov.ufsc.br/portal (15-3-2011).

(5) La métrica LEFIS. Ver su contenido en: http://www.egobs.org/index.php?option=com content\&ta sk=view\&id=14\&ltemid=27 (15-3-2011).

(6) Ver http://www.egobs.org/index.php?option=com_conte nt\&task=view\&id=21\&ltemid=64 y http://www.egobs.org lindex.php?option=com_content\&task=view\&id=26\&ltem id=69 (15-3-2011). 
(7) Ver los mapas en http://www.egov.ufsc.br/mapas/ inicio.seam (15-3-2011)

(8) El plan de gobierno electrónico de la Unión Europea para el período 2011-15 está regulado en: Communication from the Commission to the European Parliament, the Council, the European Economic and Social Committee and the Committee of the Re-gions, The European eGovernment Action Plan 2011-2015. Harnessing ICT to promote smart, sustainable \& innovative Government, COM/2010/0743 final: http://eur-lex.europa.eu/LexUri Serv/LexUriServ.do?uri=CELEX:DKEY=548503:EN:NOT (15-3-2011).

(9) Sobre servicios administrativos en línea para ciudadanos en España véase: http://www.060.es/060/appman ager/portal/desktop/page/ciudadanosHome (15-3-2011)

(10) Se toma como referencia el funcionamiento del sistema español LEXNET: "sistema informático de telecomunicaciones Lexnet para la presentación de escritos y documen-tos, el traslado de copias y la realización de actos de comunicación procesal por medios telemáticos", regulado por el Real Decreto 84/2007, de 26 de enero. La página web del sistema LEXNET es https://infolex net.justicia.es/ (15-3-2011).

\section{Referencias}

Carbonell, M. (2008). Constitution's functionality and social rights: outline of some problems. // Estudios Constitucionales. 6/2 (2008) 43-71.

Christou, G.; Simpson, S. (2009) New governance, the internet, and country code Top-level domains in Europe. // Governance. 22:4 (2009) 599-624.

Galindo, F., Justicia, gobernanza y legalidad. // Seqûencia. 55 (2007) 29-64.

Galindo, F.; García Marco, J.; Lasala, P. (2006). EGOBS: an International Electronic Government Observatory, Initial Results. // Andersen, K. V.; et al. (eds.) Electronic Government: Proceedings of the Fifth International EGOV Conference, 4-8 Septiembre 2006, Polonia. Universitätsverlag Rudolf Trauner, Linz. 317-324.

Katz, E. D. Engineering the Endgame. // Michigan Law Review. 109/3 ( 2010) 349-386.

Maturana, H. Self-consciousness: How? When? Where?. // Constructivist Foundations. 1:3 (2006) 91-102.

Mezzaroba, O.; Rover, A. J. (2009). A urna eletrônica: sua contribuição para o aperfeiçoamento da democracia representativa partidária brasileira. // Galindo, F.; Rover, A. J., (eds.). Derecho, gobernanza y tecnologías de la información en la sociedad del conocimiento. Zaragoza: Prensas Universitarias. 63-73. (LEFIS Series; 7). 
